\title{
The Feasibility of a Laparoscopic Approach to Reoperative Ileoanal J-pouch Surgery
}

\author{
Petrucci, AM ${ }^{1}$, Chadi, S2 , Mizrahi, I ${ }^{1}$, Wexner, $\mathrm{SD}^{1}$
}

${ }^{1}$ Cleveland Clinic Florida, Department of Colorectal Surgery, Weston, FL, USA

2 Toronto Western Hospital, University Health Network, Dept. of Surgery, Toronto, ON, Canada

\section{Background and Aim}

No previous series have demonstrated the feasibility of a laparoscopic approach for reoperative pouch surgery. The objective of this single surgeon cohort was to establish the feasibility of a laparoscopic approach in patients presenting to a tertiary care center with pouch-related conditions necessitating reoperation for repair, revision or excision. This series is an updated, unpublished pilot series presented as a poster at the Society of American Gastrointestinal and Endoscopic Surgeons (SAGES) in Boston, MA, on March 17, 2016.

\section{Methods}

Patient demographics and outcomes were retrospectively collected from an IRB approved prospective database and from IRB approved chart review. All procedures were performed between 2013 and 2016 by a single surgeon (SDW) with an extensive expertise in both laparoscopic and reoperative pouch surgery.

\section{Results}

- 14 laparoscopic reoperative pouch procedures were performed on 11 patients.

\section{Table 1: Demographics}

\section{Preo-op diagnosis}

- Mucosal Ulcerative Colitis

- Familial Adenomatous Polyposis

Mean age, years (range)

Mean BMI, kg/m (range)

Days from initial pouch surgery (range)

$1007(2-12562)$

Table 2: Indications for Surgery

\begin{tabular}{|c|c|}
\hline & $\mathbf{N = 1 4}$ \\
\hline Pouch volvulus with obstruction & 1 \\
\hline Pouch related fistulas & 5 \\
\hline Pouch-anastomotic dehiscence & 1 \\
\hline High-grade dysplasia & 1 \\
\hline Carcinoma & 1 \\
\hline Chronic pouchitis & 2 \\
\hline Pouch-anal anastomotic stricture & 1 \\
\hline Fecal incontinence & 2 \\
\hline
\end{tabular}

Table 3: Surgical variables

\begin{tabular}{|l|c|}
\hline Initial Surgical approach & \\
\hline - Laparoscopy & $9 / 11$ \\
\hline Procedures (total) & 14 \\
\hline - Laparoscopic pouch revision or repair & $7 / 14$ \\
\hline - Laparoscopic pouch excision & $7 / 14$ \\
\hline Mean OR time, min (range) & $273(107-430)$ \\
\hline Conversions & 2 \\
\hline - Pre-emptive, secondary to: & 1 \\
\hline \multicolumn{2}{|c|}{ Extensive pelvic fibrosis } \\
\hline Dense intra-abdominal adhesions & 1 \\
\hline Reactive & 0 \\
\hline
\end{tabular}

\section{Table 4: Post-operative variables}

\begin{tabular}{|l|c|}
\hline Post-operative morbidity & $\mathbf{4}$ \\
\hline - Wound infection & 1 \\
\hline - Pelvic fluid collections* & 2 \\
\hline - Intra- abdominal collection* & 1 \\
\hline Mean length of stay, days (range) & $9.5(3-24)$ \\
\hline
\end{tabular}

*All collections were successfully drained through percutaneous imaging techniques

\section{Conclusion}

Our updated series demonstrates laparoscopic reoperative pouch surgery to be a feasible approach by surgeons who practice high-volume minimally invasive and pouch-related surgery. Despite long operative times, patients can still benefit from the advantages of a minimally invasive approach. 